\title{
Auswertung von SAE-Level-3-Fahrfunktionen auf europäischen Straßen
}

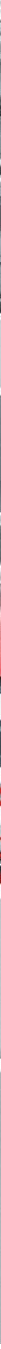

Technologien für das automatisierte Fahren müssen sicher und effizient sein, um zur Marktreife zu gelangen. Unter Beteiligung der FEV werden im EU-Forschungsprojekt L3Pilot europaweite Straßentests zu automatisierten Fahrfunktionen vorgenommen und in großem Maßstab ausgewertet. Das FEV-Testfahrzeug, seine Sensorik und erste Erfahrungen beim Datensammeln mit diesem Fahrzeug auf öffentlichen Straßen präsentiert dieser Beitrag.

Das europäische Forschungsprojekt L3Pilot hat das Ziel, automatisierte Fahrfunktionen zu testen und nachzuweisen, dass das automatisierte Fahren ein effizienter und sicherer Weg ist, den zukünftigen Transport auf den Straßen sicherzustellen. Im Projekt erfolgt eine
Konzentration auf großflächig angelegte Tests von SAE-Level-3-Funktionen sowie von einigen Level-4-Funktionen [1].

Der Schlüssel beim Testen von Systemen ist, dass man sie unterschiedlichen Konditionen aussetzt und trotzdem das Verhalten des Systems immer konsistent, verlässlich und vorhersehbar reagiert. Das ist auch der Grund, warum innerhalb des L3Pilot-Projekts die Systeme möglichst vielen Nutzern und unterschiedlichsten Verkehrsumgebungen mit konventionellen Fahrzeugen und gefährdeten Verkehrsteilnehmern ausgesetzt 


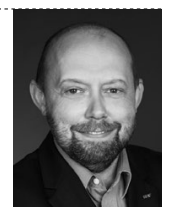

Dipl.-Ing. (FH) Markus Kremer Ist Projektleiter ADAS/AD bei der FEV Europe GmbH in Aachen.

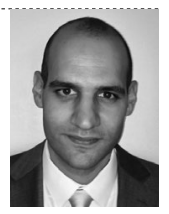

Dipl.-Ing. (FH) Khalil Hamidi ist Teamleiter ADAS/AD bei der FEV Europe GmbH in Aachen.

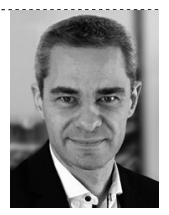

Dipl.-Ing.

Sébastien Christiaens, M. Sc. ist Abteilungsleiter ADAS/AD bei der FEV Europe GmbH in Aachen.

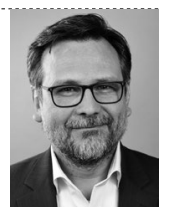

Dr. Thomas Hülshorst ist Group Vice President Intelligent Mobility and Software bei der

FEV Group in Aachen.

werden, um somit eine standardisierte europaweit gültige Testbasis für das automatisierte Fahren zu schaffen.

Im Projektkonsortium kommen Interessenvertreter aus der gesamten Wertschöpfungskette der Automobilindustrie zusammen, darunter Hersteller, Zulieferer, Universitäten, Forschungseinrichtungen, Infrastrukturbetreiber, Regierungsbehörden, Versicherungen und Nutzergruppenvertreter. Mehr als 1000 Nutzer werden ungefähr 100 Fahrzeuge in zehn europäischen Ländern testen, BILD 1. Das Projekt startete 2017, dauert 48 Monate und beinhaltet 18 Monate an Fahrtests auf öffentlichen Straßen.

\section{FEV-DEMONSTRATORFAHRZEUG MIT SENSORIK}

Für den Feldversuch nutzt FEV ein eigens entwickeltes Demonstratorfahrzeug, das mit einem Satz an Sensoren sowie mit adäquaten Steuergeräten und Datenloggern ausgestattet ist, BILD 2. Mithilfe einer Schnittstelle zum Antriebsstrang, den Bremsen und der Lenkung des Fahrzeugs können Funktionen entwickelt werden, die den Fahrer während der Fahrt unterstützen oder sogar bei einigen spezifischen Fahrsituationen ersetzen können.

Neben den Sensoren, die für die Umgebungserfassung rund um das Fahr- zeug benutzt werden, ist das Fahrzeug auch mit Fahrzeuginnenraumsensoren ausgestattet, die es erlauben, die Aktivitäten des Fahrers während der Fahrt zu erfassen. Hierzu gehören auch solche Tätigkeiten, die nicht aktiv zum Führen des Fahrzeugs erforderlich sind, oder, wenn das System den Fahrer dazu auffordert, die Kontrolle wieder zu übernehmen. Diese Sensoren erfassen simultan das Gesicht, die Hände und die Füße des Fahrers. Dies ermöglicht dann eine detaillierte Analyse der Interaktion des Fahrers mit dem automatisierten Fahrsystem in unterschiedlichsten Fahrsituationen. 


\section{STAUCHAUFFEUR}

Im Rahmen des L3Pilot-Projekts werden die Anwendungen Parkchauffeur, Stauchauffeur, Autobahnchauffeur und Stadtchauffeur getestet. Das FEV-Fahrzeug ist mit einem eigens entwickelten Stauchauffeursystem ausgestattet, das die Kontrolle des Fahrzeugs in einem Stau bis zu einer Geschwindigkeit von $60 \mathrm{~km} / \mathrm{h}$ übernehmen kann. Wenn das Fahrzeug auf einer qualifizierten Straße fährt und zusätzlich ein langsam fahrendes Fahrzeug vor dem eigenen (Ego-)Fahrzeug erkannt wird, was auf einen Stau schließen lässt, wird dem Fahrer angezeigt, dass der Stauchauffeur aktiviert werden kann. Wenn der Fahrer das System aktiviert, wird das Fahrzeug die Fahrfunktion übernehmen und innerhalb des eigenen Fahrstreifens mit genügend Sicherheitsabstand zum Vorderfahrzeug bleiben, ohne dass ein Eingriff des Fahrers oder eine Übernahme erforderlich ist. Dies gilt solange, bis entweder der Fahrer den Stauchauffeur durch eine Übernahme die Fahrfunktion deaktiviert oder bis das System das Ende des Staus oder das Ende der Autobahn erkennt. Wann immer das System das Stauende oder das Ende der Autobahn registriert, wird dem Fahrer durch ein akustisches und visuelles Signal mitgeteilt, dass er die Kontrolle wieder übernehmen muss.

Die Dauer der Fahrtests ist auf $200 \mathrm{~h}$ angesetzt, die das Fahrzeug im automa-

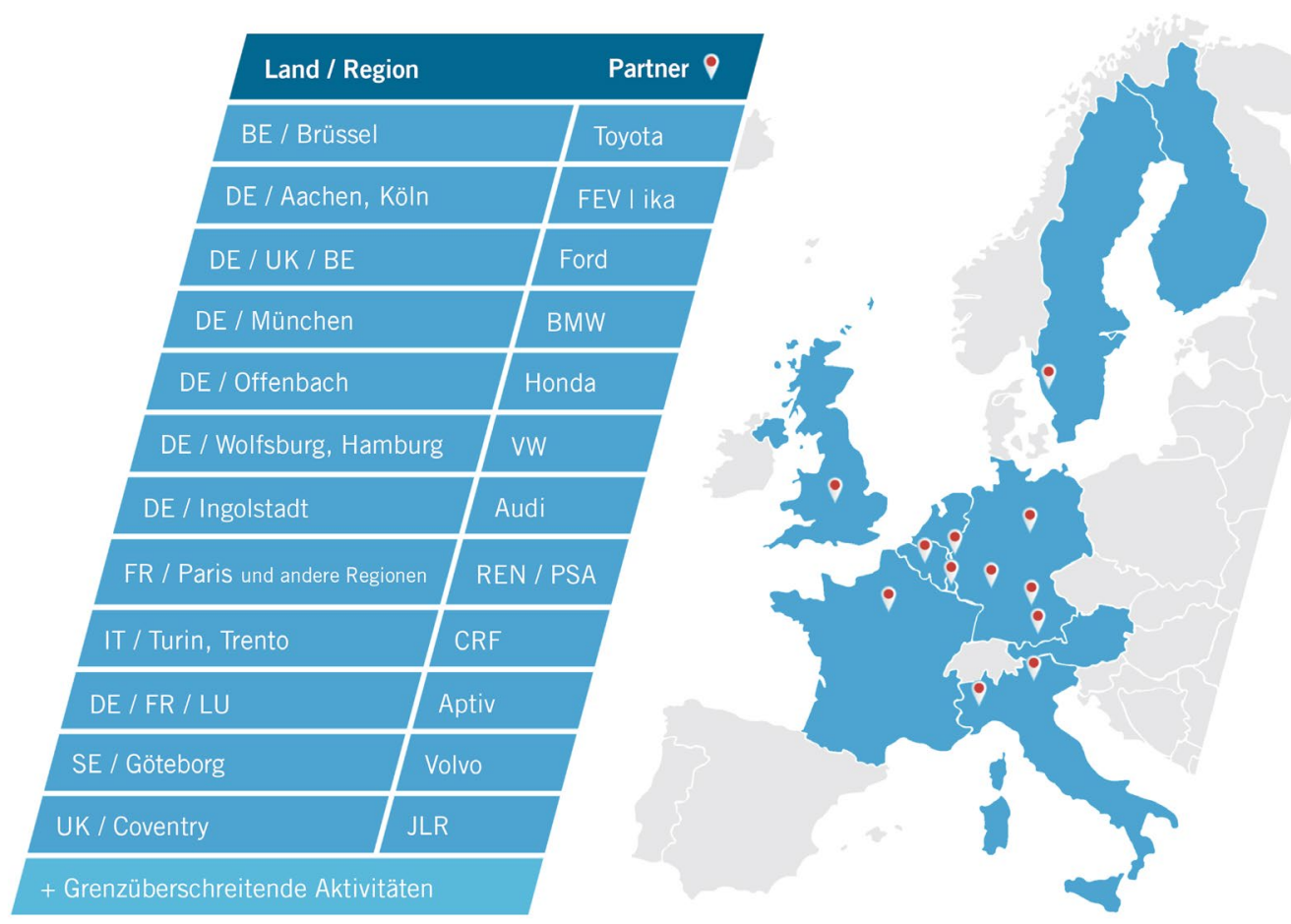

BILD 1 Übersicht der Länder und Automobilhersteller, die am L3Pilot-Projekt teilnehmen (C) L3Pilot Consortium)

tischen Stauchauffeurfahrmodus auf öffentlichen Straßen absolviert. Während dieser Testphase werden in Deutschland 10 bis 15 unterschiedliche Fahrer den Stauchauffeur auf Autobahnen zwischen Aachen, Köln und Düsseldorf testen, BILD 3, und Daten für die nachfolgende Analysephase des Projekts sammeln.

\section{DATENSAMMLUNG UND ANALYSE}

Der Auswerteprozess beinhaltet sowohl die Auswertung der subjektiven Daten, die von den teilnehmenden Fahrern mithilfe von Fragebögen gesammelt werden, als auch die Analyse von objektiven Daten, die im Fahrzeug zum Beispiel durch Aufzeichnen der internen Kommu-

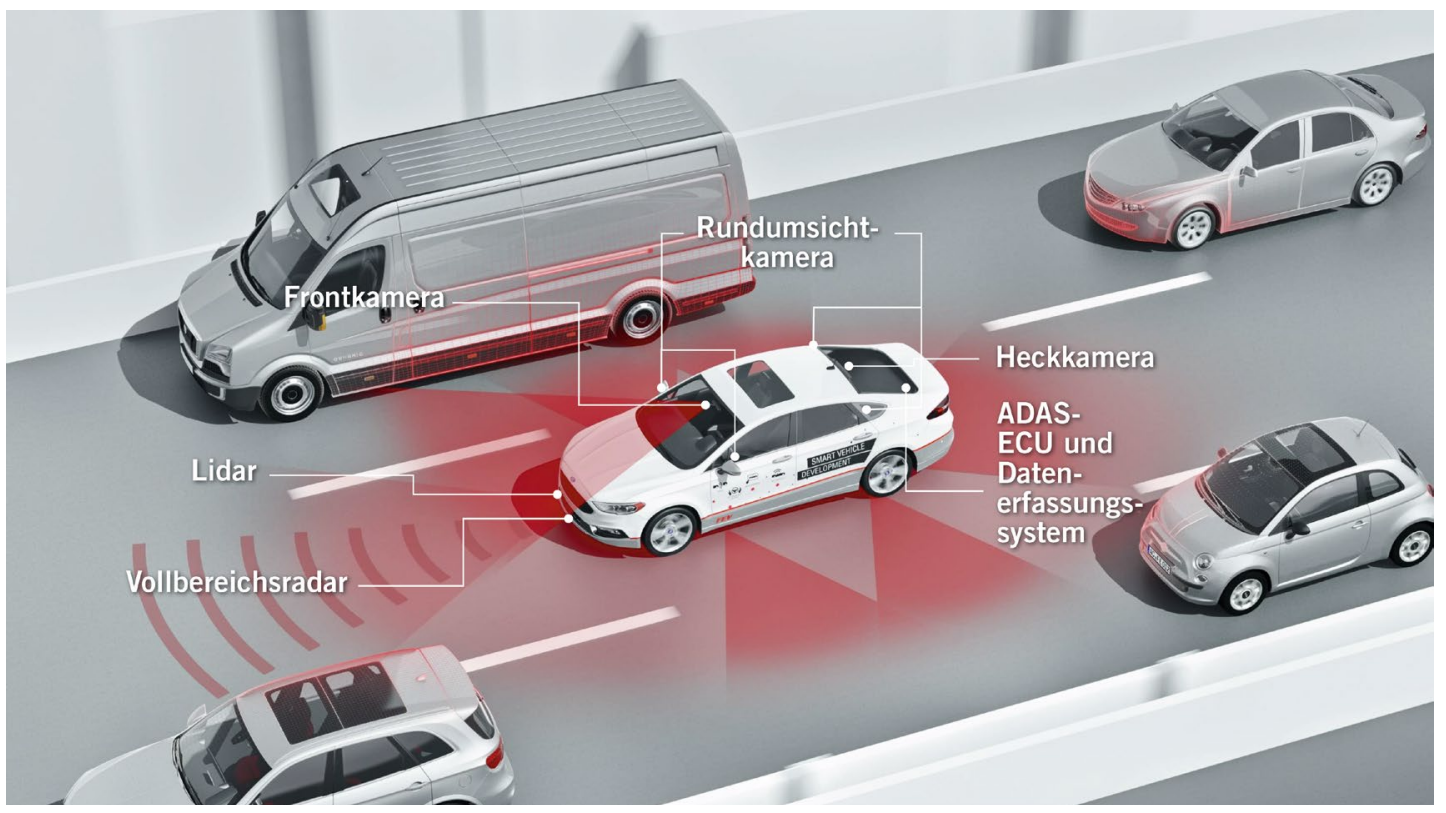

BILD 2 Übersicht über die im Demonstratorfahrzeug eingesetzten Hauptsensortechnologien (C FEV) 


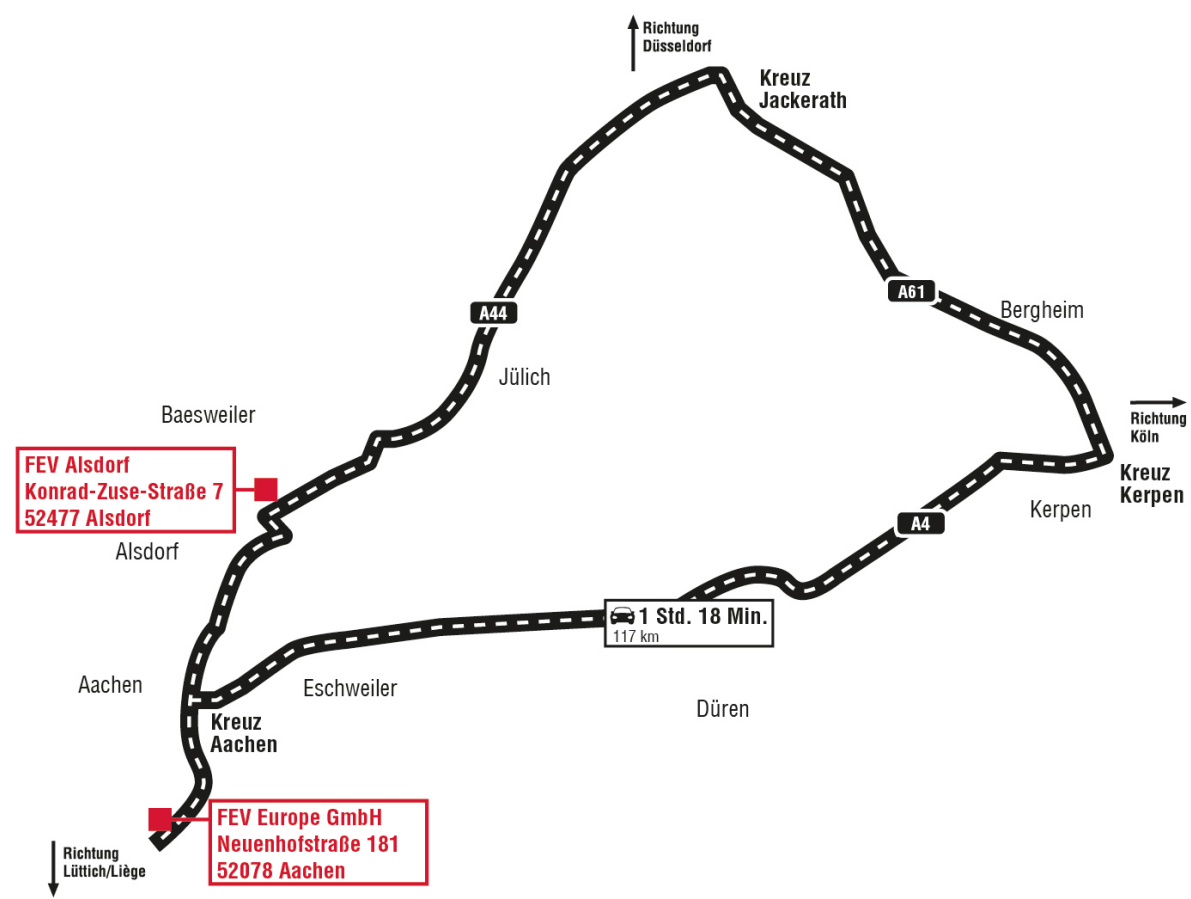

BILD 3 Ausgewählte Route für die Fahrtests mit dem SAE-Level-3-Demonstratorfahrzeugs von FEV (C FEV)

nikationswege, etwa des CAN-Busses, im Fahrzeug erfasst werden. Diese gesamten im Projekt gesammelten Daten werden zusammengeführt. Basierend auf den objektiven und subjektiven Daten der einzelnen Standorte werden die automatisierten Fahrfunktionen in Bezug auf technische und nutzerbezogene Fragestellungen ausgewertet.
Für die Datensammlung des Fahrzeugs werden zwei Datenlogger kombiniert, BILD 4. Ein Logger - im folgenden ICU genannt - ist über das Mobilfunknetzwerk mit Cloudservern verbunden und schickt kontinuierlich vorselektierte Daten vom Fahrzeug in die Cloud. Der zweite Datenlogger sammelt alle Rohund Objektdaten von Sensoren sowie von Aktuatoren und vom Steuerungskontrollsystem. Diese Daten werden auf einer Festplatte im Fahrzeug gespeichert.

Die Analyse der Daten beginnt sofort, wenn sie vom Mobilfunknetzwerk durch die ICU an die Cloud übermittelt wurden. Sobald die Daten auf dem Server zur Verfügung stehen, werden sie zeitlich synchronisiert und in das L3Pilot-spezifische Common Data Format (CDF) [5] konvertiert, das auf dem Open-source Hierarchical Data Format (HDF5) basiert.

Sobald die Daten aufbereitet sind, werden sie an den ausgewählten Analysepartner im Konsortium übermittelt, der diese dann durch die umfassende Datenaufbereitungsumgebung schickt. Diese wurde basierend auf Werkzeugen und Erfahrungen aus den Vorgängerprojekten euroFOT [6] und AdaptIVe [7] entwickelt. Unter anderen Auswerteroutinen laufen Programme, die bestimmte, im Rahmen des Projekts definierte Szenarien automatisch erkennen. Die erkannten Szenarien werden automatisch in den Datenbanken annotiert.

Ziel dieses szenariobasierten Ansatzes ist es, das Verhalten des automatisierten Fahrsystems aus Sicht des technischen und verkehrstechnischen Standpunkts zu analysieren. Um dieses Ziel zu erreichen, werden sowohl abgeleitete Maßnahmen (AM), als auch Leistungsindikatoren (LI) aus den Daten, basierend auf den Szenarien, durch den Analysepartner abgeleitet. [8]

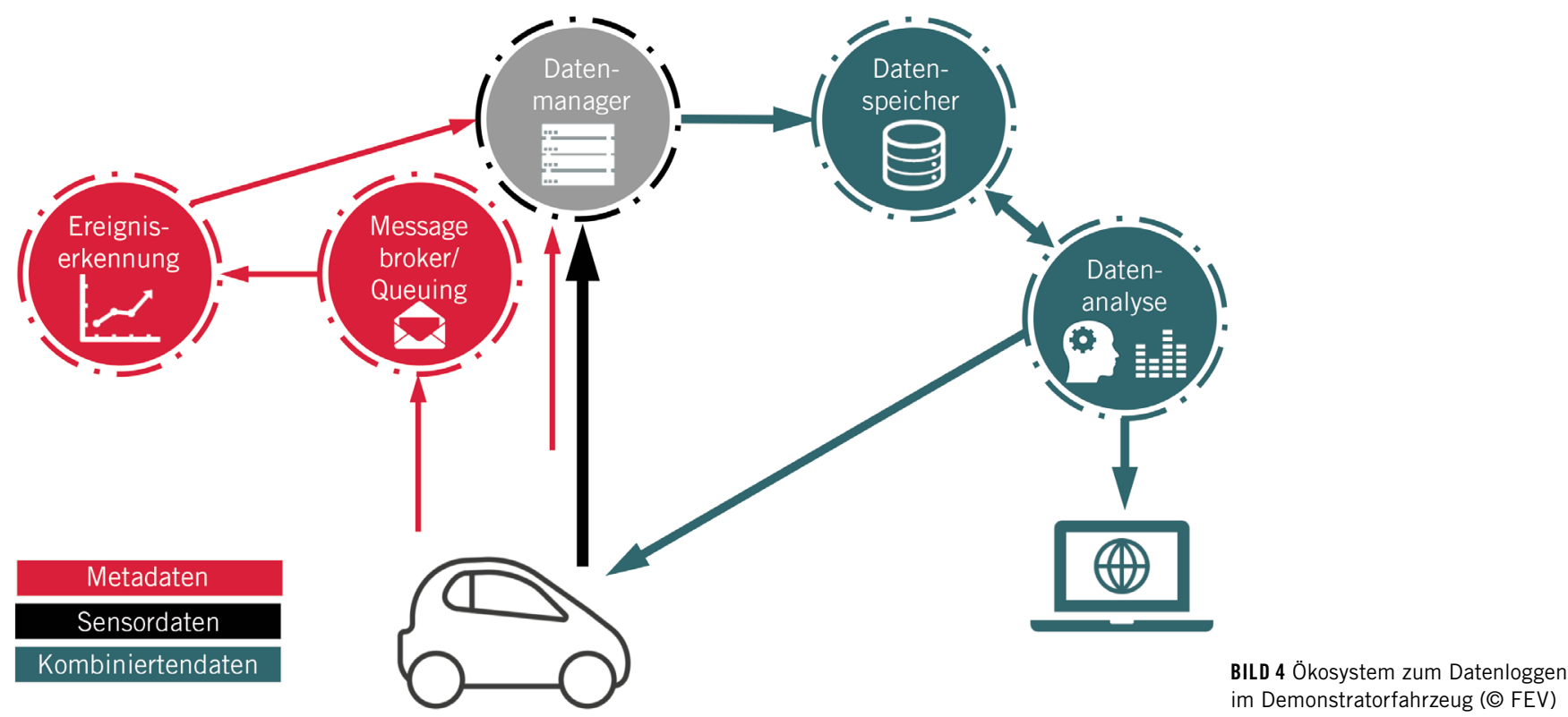




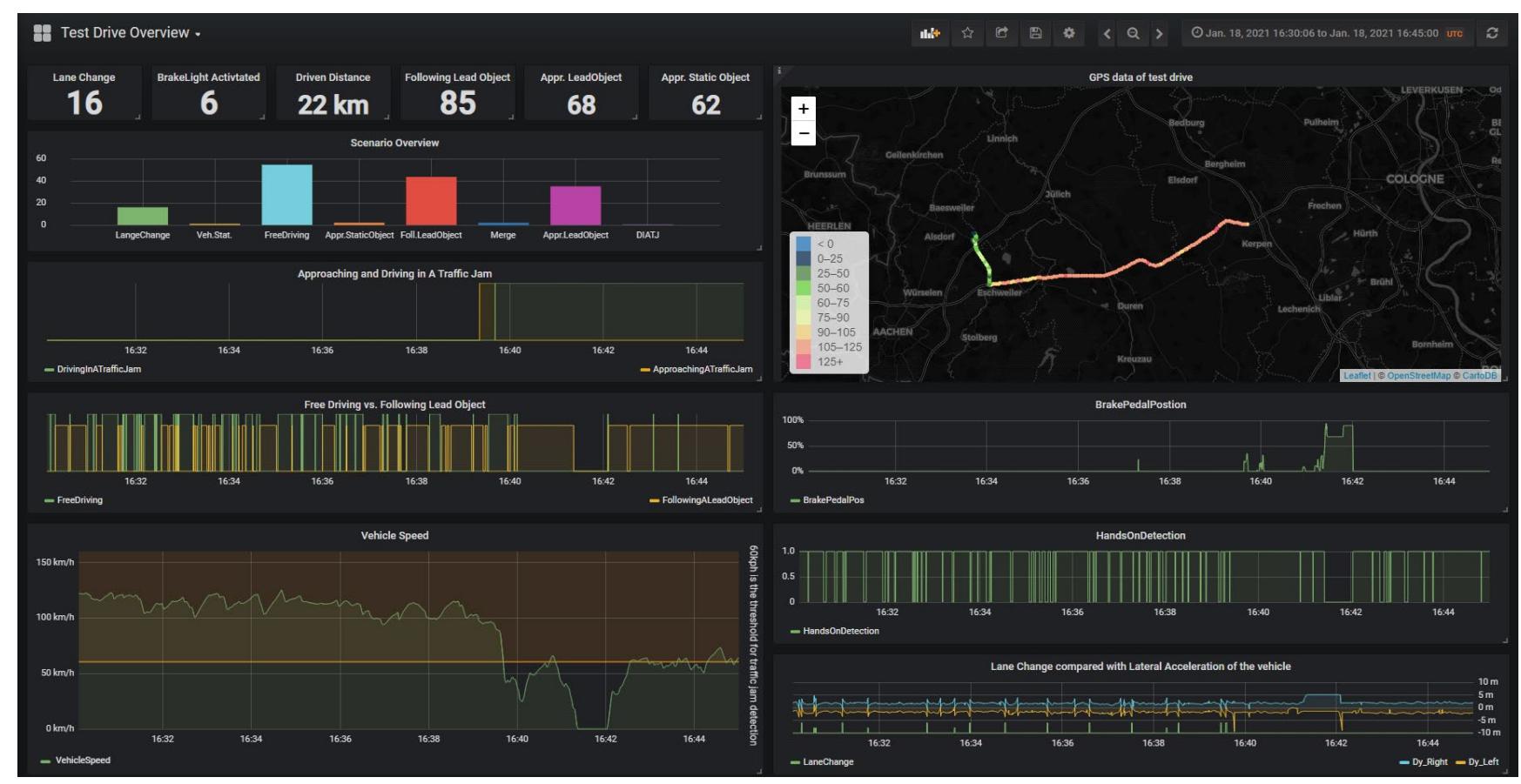

BILD 5 Übersicht einer Testfahrt (@ FEV)

Zum Beispiel sind die AM oder LI so definiert worden, dass eine Analyse dahin gehend möglich ist, ob das Fahrzeug einen sicheren Abstand zum Vorderfahrzeug halten kann oder wie sanft sich das Fahrzeug einem stehenden Fahrzeug nähert und später wieder losfährt. Die Anzahl von Fahrerübernahmen und die Häufigkeit dieser Anfragen sind weitere Themen, die im Detail analysiert werden. Um die Analyse der LI zu vereinfachen, wurde eine webbasierte Anzeige erstellt, die die Auswertung der Analysen aller LI darstellen kann, BILD 5.

Die gesamten während des Projekts gesammelten Daten werden dann durch die Analysepartner so analysiert und aggregiert, dass weitere generelle Informationen gewonnen werden können.

\section{ZUSAMMENFASSUNG UND AUSBLICK}

Die Durchführung umfangreicher Fahrversuche im realen Straßenverkehr ist ein wichtiger Schritt zur Entwicklung und Validierung der für autonomes Fahren erforderlichen Systeme. Die Messungen erzeugen wertvolle Daten und Informationen, die zur Bewertung und Weiterentwicklung autonomer Fahrzeuge essenziell sind. Das L3Pilot-Projekt wird auch zusätzliche Informationen über die Nut- zerakzeptanz von automatisierten Fahrsystemen liefern. Dazu gehören Fahrund Reiseverhalten, aber auch die Einflüsse auf den Verkehr und die Gesellschaft. Das Projekt ebnet den Weg für großangelegte Feldversuche von seriennahen autonomen Fahrzeugen auf öffentlichen Straßen und erstellt ein solides Rahmenwerk für die Harmonisierung dieser Aktivitäten zwischen den verschiedenen Automobilherstellern und anderen Partnern der Automobilindustrie.

Aufgrund der aktuellen Covid-19-Pandemie konnte bis zur Abgabe dieses Artikels nur ein kleiner Teil der ursprünglich geplanten Testdaten eingefahren werden. Die bisher gewonnenen Daten reichten noch nicht aus, um abschließende Ergebnisse oder Schlüsse zu ziehen. Der weitere Verlauf der Tests und des Projekts wird unter anderem auf der Internetseite des Projekts zur Verfügung gestellt [1].

\section{LITERATURHINWEISE}

[1] EICT (Hrsg.): L3Pilot-Projekt. Online: www.I3pilot.eu, aufgerufen: 8. September 2020 [2] SAE: Taxonomy and definitions for terms related to on-road motor vehicle automated driving systems. Standard No. J3016, SAE International, 2016

[3] Kreutz, M.; Macke D.; Kremer, M.; Hülshorst, T.: Effiziente Datenverwaltung mittels vernetzter Logger. In: ATZelektronik16 (2021), Nr. 7-8

[4] EICT (Hrsg.): Deliverable D4.1 - Description and Taxonomy of Automated Driving Functions L3Pilot -
Driving Automation. Online: https:///3pilot.eu/fileadmin/user_upload/Downloads/Deliverables/L3Pilot-SP4-D4.1-Description_and_taxonomy_of_AD_ functions-v2.0_for_website.pdf, aufgerufen: 16. April 2021

[5] Hiller, J.; Svanberg, E.; Koskinen, S.; Bellotti, F.; Osman, N.: The L3Pilot Common Data Format Enabling Efficient Automated Driving Data Analysis. In: 26 th International Technical Conference on the Enhanced Safety of Vehicles (ESV), Eindhoven, 2019

[6] EICT (Hrsg.): AdaptIVe-Projekt. Online: www. adaptive-ip.eu, aufgerufen: 12. Oktober 2020 [7] EICT (Hrsg.): euroFOT-Projekt. Online: www. eurofot-ip.eu, aufgerufen: 12. Oktober 2020 [8] Hiller, J.; Koskinen, S.; Berta, R.; Osman, N.; Nagy, B.; Bellotti, F.; Rahman, A.; Svanberg, E.; Weber, H.; Arnold, E.; Dianati, M.; de Gloria, A.: The L3Pilot Data Management Toolchain for a Level 3 Vehicle Automation Pilot. In: Electronics 5 (2020), Nr. 9, S. 809

\section{DANKE}

Das L3Pilot-Projekt wird durch das Forschungs- und Innovationsprogramm Horizon 2020 der Europäischen Union unter der Vereinbarung mit der Nummer 723051 gefördert. Die Autoren bedanken sich bei allen Partnern des Konsortiums für die Kooperation und die wertvolle Zusammenarbeit. Verantwortlich für die geteilten Informationen und Ansichten sind allein die Autoren. 


\section{Die besten Seiten der Branche.}

Der AVK Composites-Navigator 2021

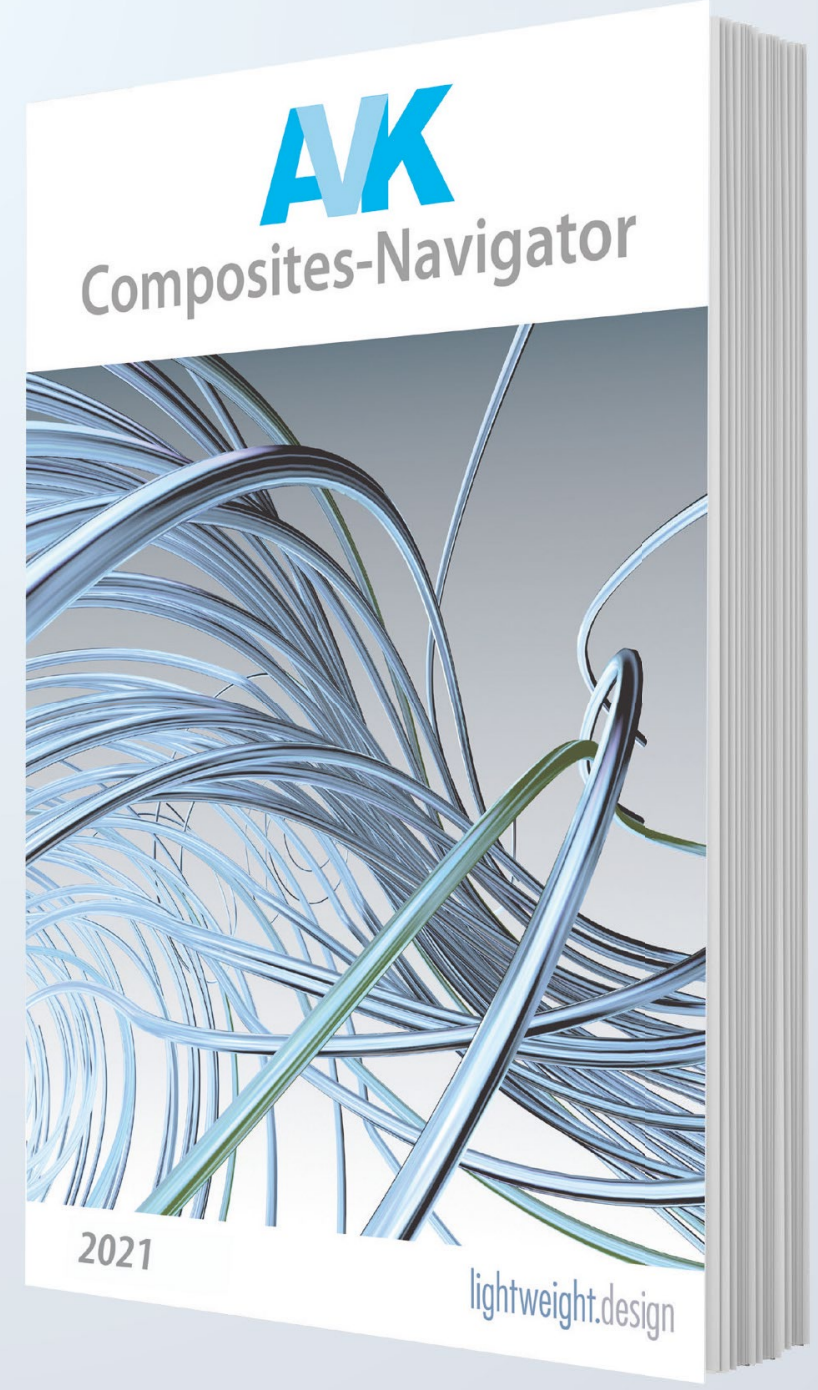

SICHERN SIE SICH

IHREN PLATZ IM

HANDBUCH

Hier werden Sie gefunden!

Im September 2021 erscheint wieder die deutschsprachige Ausgabe des AVK Composites-Navigator - ein umfassendes Nachschlagewerk zu Unternehmen und Institutionen entlang der Wertschöpfungskette von Bauteilen aus faserverstärkten Kunststoffen sowie technischen Duroplasten. Nutzen Sie die Gelegenheit in diesem Handbuch aus der Branche und für die Branche präsent zu sein. Der in Kooperation mit lightweight.design erscheinende AVK Composites-Navigator erscheint auch als E-Book mit effizienten und komfortablen Suchfunktionen.

Ein unerlässliches Tool für Profis, die suchen - und Profis, die gefunden werden wollen. 